\title{
A CubeSAT Payload for In-Situ Monitoring of Pentacene Degradation due to Atomic Oxygen Etching in LEO
}

\author{
Sergi Gorreta ${ }^{\mathrm{a}}$, Joan Pons-Nin ${ }^{\mathrm{a}, *}$, Gema López $^{\mathrm{a}}$, Eduard Figueras $^{\mathrm{b}}$, Roger Jove-Casulleras ${ }^{\mathrm{c}}$, Carles Araguz $^{\mathrm{c}}$, Pol Via $^{\mathrm{c}}$, \\ Adriano Camps ${ }^{\mathrm{c}}$, Manuel Dominguez-Pumar ${ }^{\mathrm{a}}$ \\ ${ }^{a}$ Micro and Nano Technologies Research Group, Electronic Engineering Department, \\ Technical University of Catalonia (UPC), Jordi Girona 1-3, 08034 Barcelona, Spain \\ ${ }^{b}$ Institut of Microelectronics of Barcelona (IMB-CNM. CSIC), Bellaterra-Barcelona, Spain \\ ${ }^{c}$ Remote Sensing Laboratory, Signal Theory and Communications Department, \\ Technical University of Catalonia (UPC), Jordi Girona 1-3, 08034 Barcelona, Spain
}

\begin{abstract}
This paper reports and discusses the design and ground tests of a CubeSat payload which allows to measure, in-situ and in real time, the degradation of a polymer of electronic interest due to atomic oxygen etching in LEO. It provides real-time information on how the degradation occurs, eliminating the need to work with samples recovered once the mission has finished. The polymer, TIPS-Pentacene, is deposited on the surface of a microelectromechanical (MEMS) cantilever, which works as a resonator embedded in a Pulsed Digital Oscillator circuit. The mass losses in the polymer due to atomic oxygen corrosion produce variations in the resonant frequency of the MEMS, which is continuously sensed by the circuit and transmitted to the ground. This way, polymer mass losses around $10^{-12} \mathrm{Kg}$ can be detected during the mission. The payload is a part of the ${ }^{3} \mathrm{Cat}-1$ mission, a nano-satellite aimed at carrying out several scientific experiments.
\end{abstract}

Keywords: nano-satellites, MEMS, atomic oxygen, pulsed digital oscillators, polymer degradation

\section{Introduction}

Environmental conditions found in space missions include aggressive factors such as ionizing, ultra-violet and electromagnetic radiation, atomic oxygen, large temperature variation, high vacuum, space debris, etc. Accordingly, the study of the degradation effects of such factors on materials to be used in aerospace applications is always a hot topic. In particular, atomic oxygen $(\mathrm{AO})$ is considered as the most serious hazard to spacecraft materials in Low Earth Orbit (LEO) [1, 2]. AO is produced from dissociation of molecular oxygen due to solar radiation at altitudes between 180 and $650 \mathrm{~km}$. Chemical reaction of $\mathrm{AO}$ with polymer, carbon and metal surfaces may produce oxides which can be either volatile or mechanically fragile, thus resulting in corrosion and mass loss.

Corrosion of polymers due to AO exposure in LEO was first observed in early shuttle missions, where the Kapton protection of the cameras was found textured and thinned after the mission [3]. The first evaluation of a variety of materials after long time LEO exposure was performed in the Long Duration Exposure Facility [4], but extensive experimental data was provided later by the MISSE (Materials International Space Station Experiment) PEACE (Polymers Erosion And Contamination Experiment) ex-

\footnotetext{
*Corresponding author: e-mail: joan.pons@upc.edu address: Jordi Girona 1-3, mòdul C4, 08034 Barcelona, Spain.
}

periments. There, samples corresponding to 41 different polymers were placed outside the International Space Station, then exposed to the LEO environment during nearly four years and analized on the ground after retrieval. MISSE PEACE data allowed to characterize AO erosion yields and to develop models and prediction tools for a variety of polymers [5, 6 ]. Moreover, significant differences between space degradation and that obtained in ground facilities (where experiments are performed in accelerated conditions to reduce experiment duration) have been explained by synergistic factors found in LEO [7, 8].

These studies about AO polymer corrosion in LEO use data obtained after mission completion or from ground laboratory facilities, and they focus on polymers mainly used for structural or protection purposes outside the spacecraft. Nevertheless, up to now no studies are based on data obtained while the mission is still being carried out or concerning materials to be used inside the spacecraft. Additionally, in recent years nano-satellites have proved to be excellent platforms for performing on-board technology tests or scientific experiments, e.g. see [9, 10, 11]. According to all this, this paper discusses the design and ground tests of a CubeSat payload that measures in-situ the AO corrosion of a polymer of electronic interest. This payload is a part of the ${ }^{3}$ Cat- 1 mission, a single-unit nano-satellite developed at the Technical University of Catalonia (UPC) following the CubeSat standard and aimed at carrying out scientific experiments and performing technology demon- 
strations [12]. ${ }^{3}$ Cat-1 launching is scheduled for the second half of 2016 and it will be deployed in a 550-650 Km elliptical polar orbit. A key aspect is that, unlike the cases cited above, the experimental results are transmitted to the ground during the mission and thus payload retrieval is unnecessary.

Pentacene is a polymer of high interest in electronics that exhibits sensitivity to AO etching [13, 14]. A specific variant, Triisopropylsilylethinyl Pentacene, commonly known as TIPS-Pentacene, was chosen for this application because of its excellent processing properties, which include solubility in several common organic solvents and good ambient stability [15]. Since it provides mobilities comparable to those of amorphous silicon, this is a very promising material for new devices, such as Organic FieldEffect Transistors (OFETs) on flexible substrates.

On the other hand, the transduction mechanism used in the payload is mass sensing. The mechanical oscillation frequency of a microelectromechanical (MEMS) cantilever with the Pentacene layer deposited on its top surface depends on the current mass of such layer, then mass losses due to AO corrosion imply oscillation frequency changes. MEMS oscillation is excited and measured with a Pulsed Digital Oscillator (PDO) 16]. PDOs are sampled non linear circuits with digital output that can be used in a variety of MEMS-based frequency sensing applications, such as sensors for Volatile Organic Compounds [17] or in-situ vacuum testing for MEMS packages [18].

This paper is organized as follows. Section 2 describes the key features of PDO structures with MEMS used as mass sensors. The section starts introducing the main properties of PDOs obtained from a linear analysis approach. Next, the non-linearity effects of PDO architectures that may affect the sensor behaviour are discussed. Finally, strategies to excite different mechanical resonances in the MEMS are introduced. This enables sensing at different frequencies with the same PDO structure. Section 3 describes the ${ }^{3}$ Cat- 1 mission and the payload hardware, including the MEMS used, the circuitry and the measurement protocol designed for the mission. It is shown that mass variations in the order of $0.5 \mathrm{ng}$ can be detected with the payload. Finally, the experimental results obtained in ground tests before and after the polymer deposition are discussed in Section 4.

\section{Mass sensing with PDOs}

This section discusses the properties of PDOs making them a very interesting option for frequency-based sensing applications. To this effect, consider as reference the PDO topology shown in Figure 1. It works as follows: the position of the MEMS resonator is compared with its rest position at each sampling time, and depending on the result a short force pulse of values $\{+F,-F\}$ or $\{+F, 0\}$ is applied to the resonator after $L$ sampling periods. This way, selfsustained oscillations can be excited in the MEMS. This circuit provides built-in analog to digital conversion, being

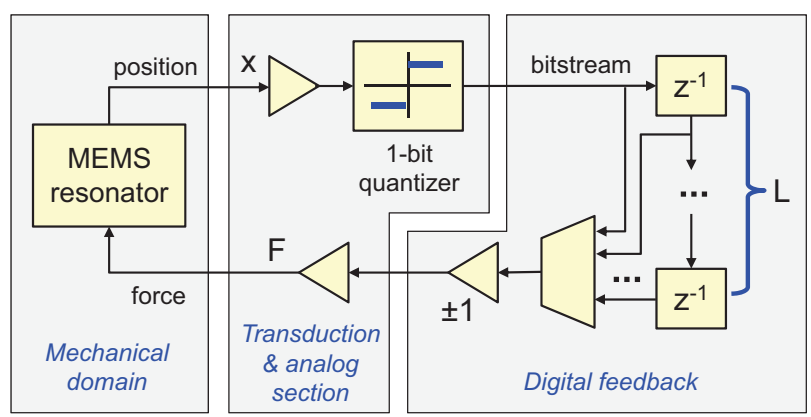

Figure 1: PDO architecture. It includes a MEMS resonator, position (or velocity) sensing, a 1-bit quantizer, a digital feedback loop with $L$ delays and a force transducer.

the digital output the sequence, or bitstream, produced in the feedback loop. Due to the presence of the 1-bit quantizer, PDOs are non linear circuits and therefore they can exhibit complex dynamics. Accordingly, in this chapter a linear approach is first used to introduce the main features of PDOs and thereafter non linear effects are discussed.

\subsection{Main features of 'linearized' PDOs}

Consider a suspended-moveable plate as the structure of the MEMS resonator. In a typical 1D approach the dynamics of the plate can be described by the following mass-spring-damper differential equation,

$$
m \frac{d^{2} x(t)}{d t^{2}}+b \frac{d x(t)}{d t}+k x(t)=F(t)
$$

being $F(t)$ the external force applied to the plate, $m$ the mass, $b$ the damping loss factor and $k$ the spring factor of the mechanical restoring force. The force applied by the PDO takes the form of a series of short pulses,

$$
F(t)=\Sigma_{n} b_{n} \delta\left(t-n T_{S}\right)
$$

where $T_{S}$ is the sampling period and

$$
b_{n}=\operatorname{sgn}\left(x\left((n-L) T_{S}\right)\right)
$$

is the bitstream provided by the 1-bit quantizer, after $\mathrm{L}$ delays at the sampling frequency (see Figure 1).

Let us now consider a specific PDO with a unique delay $(L=1)$. As commented above, PDOs are non linear. In order to overcome this and perform a first analysis, let us linearise the circuit by replacing the 1-bit quantizer by gaussian-white noise addition, as usually done in $\Sigma-\Delta$ related systems [19]. Now, taking into account the sampled impulse response of the MEMS extracted from eq. (11), a standard linear analysis of the oscillator loop can be performed. This analysis yields that the digital oscillation frequency provided by the bitstream is [16],

$$
f_{D}=\frac{1}{2 \pi} \operatorname{Cos}^{-1}\left(e^{-2 \pi \rho f_{R}} \cos \left(2 \pi f_{R} \sqrt{1-\rho^{2}}\right)\right)
$$

where $f_{R}=f_{0} / f_{S}$ is the normalized resonant frequency, $f_{0}$ the resonant frequency of the MEMS and $f_{S}=1 / T_{S}$ the 


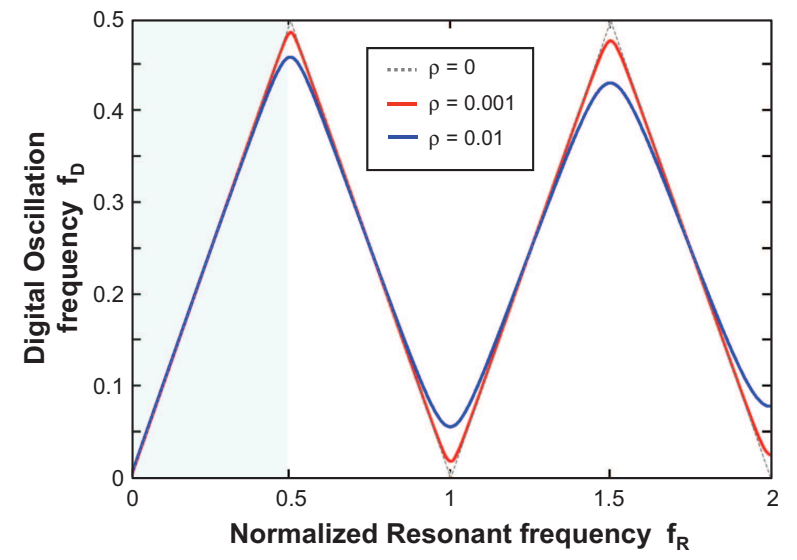

Figure 2: Digital oscillation frequency of a 'linearized' PDO versus the normalized resonant frequency for $\rho=\{0,0.01,0.1\}$. The shadowed area corresponds to oversampling conditions.

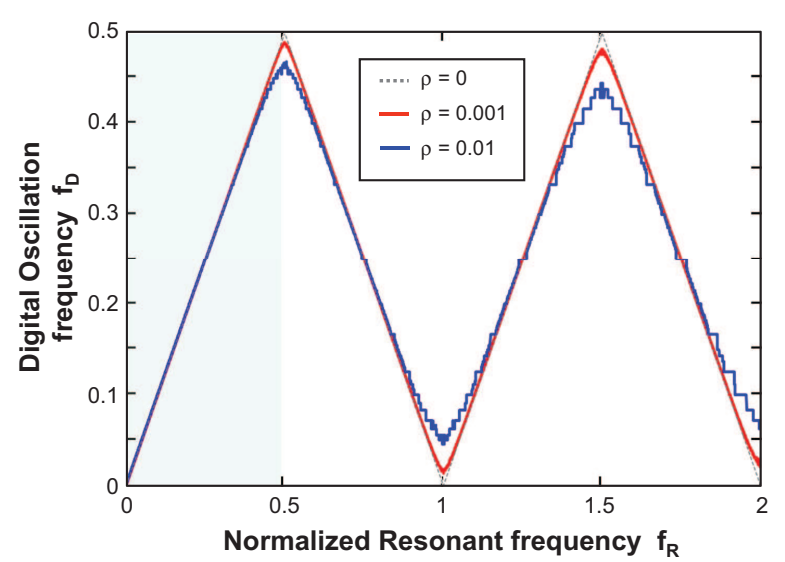

Figure 3: Discrete-time simulation results of the PDO digital oscillation frequency versus the normalized resonant frequency for $\rho=\{0,0.01,0.1\}$. The digital frequency was obtained for $10 \times 2^{10}$ different values of $f_{R}$, during $10^{7}$ clock cycles.

sampling frequency; $\rho=1 / 2 Q$ is a dimensionless damping factor and $Q$ the quality factor of the MEMS. These parameters are related to the mechanical ones of eq. (1) through,

$$
\rho=\frac{b}{2 \sqrt{k m}}, \quad f_{0}=\frac{1}{2 \pi} \sqrt{\frac{k}{m}}
$$

It is found from eq. (4) that the oscillation frequency of the PDO, $f_{O S C}$, is a function of $f_{0}, f_{S}$ and $\rho$. The relationship between $f_{D}$ and $f_{R}$ for different values of $\rho$ is plotted in Figure 2. For MEMS with no damping losses $(\rho=0)$ and normalized resonant frequencies in the range $f_{R} \leq 0.5$, it yields $f_{D}=f_{R}$, which implies PDO oscillation at the mechanical resonant frequency of the MEMS: $f_{O S C}=f_{D} \times f_{S}=f_{0}$. This 'ideal' result becomes progressively distorted for resonators with noticeable damping losses and increasing values of $f_{R}$. However, the LEO environment implies high vacuum conditions and for our devices negligible damping losses are expected there.
Equation (4) is also valid for $f_{R}>0.5$, where the Nyquist criterion is not met. That is, the PDO also works in what has been called under-sampling conditions: although it can still oscillate at frequencies $f_{O S C}$ close to $f_{0}$, the digital frequency remains confined in the range $0 \leq f_{D} \leq 0.5$, thus suffering from aliasing. As a consequence, the relation between $f_{O S C}$ and $f_{D}$ is different in each 0.5 -length segment of the $f_{R}$ axis. This relation is given by [16],

$$
f_{O S C}= \begin{cases}f_{S} \cdot\left(\frac{r}{2}+f_{D}\right), & r=\text { even } \\ f_{S} \cdot\left(\frac{r+1}{2}-f_{D}\right), & r=\text { odd }\end{cases}
$$

where $r=\left\lfloor 2 f_{R}-0.5\right\rfloor$. This is a key result, since it means that the actual oscillation frequency can always be inferred from $f_{D}$ if the segment $r$ where $f_{R}$ lies is known; this can be done through previous characterization of the MEMS allowing to obtain $f_{0}$.

Finally, let us note that eqs. (5) and (6) provide the basis for mass sensing with PDOs: when the circuit is oscillating tuned to the resonant frequency of the MEMS, mass variations produce changes in $f_{0}$ that can be detected as changes in $f_{D}$, then in $f_{O S C}$.

\subsection{Non linear effects}

Up to now, useful properties for mass sensing have been identified, but for a 'linearized' PDO. At this point it becomes necessary to check whether these properties are affected by the intrinsic non linearity of PDOs. To this effect, discrete-time simulations of the structure can be performed [16]. Results are shown in Figure 3, where the digital frequency is plotted against the normalized resonant frequency for the same cases as in Figure 2, The plots illustrate that the actual behaviour of the PDO closely follows the tendency predicted by the linear theory, but with a fractal shape. This fractal shape implies an undesired frequency-locking effect: in the plateaus, small variations of $f_{0}$ (then of $f_{R}$ ) are not detectable because they produce no changes in $f_{D}$. Anyway, this behaviour is significant only for high damping losses and deep undersampling conditions.

On the other hand, it is demonstrated in [16] that the output bitstreams $b_{n}$ produced by PDOs can be related to first-order $\Sigma-\Delta$ sequences $S D_{n}$ through a simple exclusiveOR function as follows:

$$
S D_{n}=b_{n} \oplus b_{n-1}
$$

This result provides a practical way to extract the digital frequency directly from the bitstream: $f_{D}=M / 2 N$, being $M$ the number of events counted in the bitstream within a time window of $N$ sampling periods.

Finally, let us take into account the effects caused by changing the number of delays $L$ or the sign of the feedback loop. For a given value of $L$, the practical result is that the characteristic $f_{D}\left(f_{R}\right)$ becomes divided into segments of equal length. Each segment denotes a frequency 


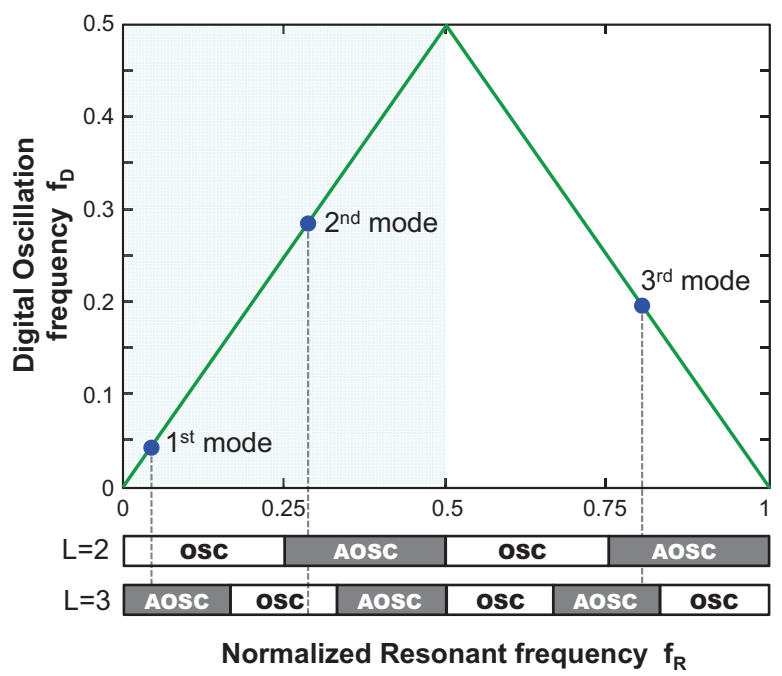

Figure 4: Example of selective excitation of two different mechanical modes for a given device. The third mode is in undersampling conditions.

range in which an oscillation is either excited in the resonator (energy is transmitted from the electrical to the mechanical domain) or reduced (energy is extracted from the mechanical domain) [20, 21]. Meanwhile, changing the sign reverses this behaviour, so that oscillation regimes become anti-oscillation and vice-versa.

This result is of great importance for mass sensing, because it allows to selectively excite different mechanical vibration modes of the MEMS by choosing appropriate values of $f_{S}, L$ and sign. This is illustrated in Figure 4 for the device used in the payload (see Section $3.2)$, which has the first three mechanical modes located at $f_{R}=\{0.042,0.283,0.801\}$ for the selected $f_{S}=110$ $\mathrm{kHz}$. Using a PDO with $L=2$ and positive sign, the second and third modes are set to anti-oscillation and the MEMS is forced to oscillate at its first mode. However, for $L=3$ the second mode is excited and the other two are set to anti-oscillation.

\section{The PDO-MEMS Payload}

\subsection{The ${ }^{3}$ Cat-1 Mission}

The ${ }^{3}$ Cat- 1 is the first project of the UPC to develop and launch a nano-satellite in compliance to the CubeSat standard. Initially being an educational project in which some of the subsystems are developed by students, the ${ }^{3}$ Cat- 1 is conceived as a cost-effective platform to perform small scientific experiments, and to demonstrate some of the new technologies that it incorporates [12]. The spacecraft integrates up to seven different payloads, five of which are aimed at carrying out scientific experiments and technology demonstrations, namely:

- A couple of coils to test Wireless Power Transfers in the presence of plasma [22],
- a Graphene Field Effect Transistor,

- a new topology of solar cells [23],

- an Energy Harvesting experiment that relies on temperature gradients in the spacecraft walls [24], and

- the PDO-MEMS sensor described here.

Two additional payloads, an optical camera and a Geiger counter, are based on commercial-off-the-shelf (COTS) components. The payloads and experiments have been designed and developed by research groups at UPC or in collaboration with international institutions. Additionally, the ${ }^{3}$ Cat- 1 is aimed to explore the capability of this class of spacecraft and to identify the need for standardized software and hardware interfaces for the development of future nano-satellite missions.

The integration of the payloads has posed significant challenges both in terms of global mass-volume and in terms of energy and subsystem management. To overcome the operability issues arising from the existence of multiple payloads with heterogeneous system constraints (e.g. execution cycles, power consumption, and data generation volume) the ${ }^{3}$ Cat-1 includes an on-board autonomous management system which is able to generate mission plans of action without human intervention. This system, mainly composed of a task scheduler, is capable of autonomously planning the payload activities that fulfil the mission goals while safely controlling the system resources, namely energy, data storage and communication bandwidth.

The development of ${ }^{3}$ Cat- 1 has been carried out at the UPC's Nano-Satellite and Small Payload Laboratory, a facility with the equipment necessary to validate and qualify nano-satellites: thermal high vacuum chamber, Sun simulator, shake table and Earth magnetic field generator. Most of the subsystems in ${ }^{3}$ Cat- 1 have been designed ad-hoc using COTS components, self-qualified in the laboratory facilities. This design choice has been motivated by integration issues (i.e. volume minimization, mechanical compatibility and reduced power consumption) and to reduce development times.

\subsection{The MEMS resonator}

The MEMS resonators used in this work are silicon suspended plates held by three cantilever arms, as the one shown in Figure 5. These devices are fabricated with the same SOI-bulk micromachining process used in [21]. The actuation mechanism is thermoelectric: cantilever deflection is caused by a force proportional to the heat dissipated in two resistor layers located in the external arms. The thermal expansion force is proportional to the square of the voltage applied to the heaters. This non-linear mechanism poses a problem in standard actuation strategies for oscillators, e.g. when sinusoidal waveforms are applied the frequency becomes doubled in the feedback loop. However, the PDO applies short pulses of voltage of constant amplitude, thus overcoming this problem. 


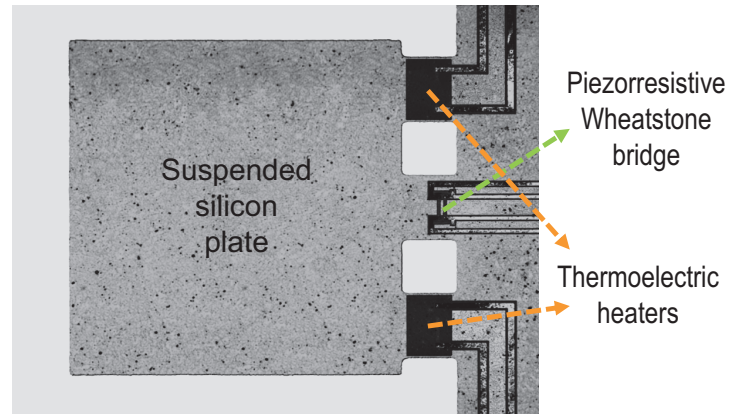

Figure 5: Structure of the MEMS resonators used in this work.

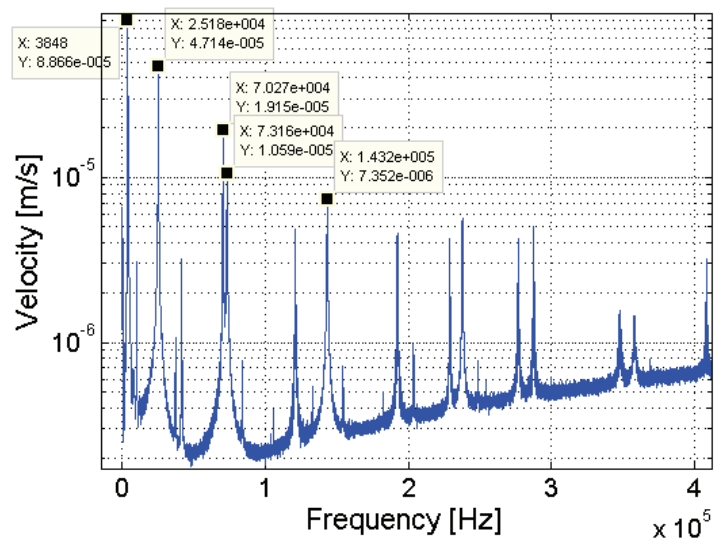

Figure 6: Mechanical vibration spectrum of the MEMS used in the payload, obtained with a laser-Doppler vibrometer after a frequency sweep. The measurement was performed in ambient pressure.

On the other hand, cantilever deflection is sensed through a piezoresistive Wheatstone bridge placed in the central arm. Due to the spatial distribution of the bridge, only movements implying longitudinal-axis deflection (that is, vertical deflection of the central arm) can be detected. Note that in PDOs only the sign of the position is needed, thus a simple comparison with a reference value (i.e. the 'rest' position) is enough to achieve this result.

The device finally selected for the payload is a squared plate of $1000 \mu m \times 1000 \mu m$ area, with $150 \mu m$ long and $200 \mu m$ wide suspension arms. The gaps between arms are $150 \mu \mathrm{m}$ wide and the thickness of the suspended structure is $5 \mu \mathrm{m}$. The mechanical vibration modes have been measured with a laser-Doppler vibrometer, with the results shown in Figure 6. First and second modes are located at $3.848 \mathrm{kHz}$ and $25.18 \mathrm{kHz}$ respectively. The experiment was performed in air, thus with noticeable damping losses.

\subsection{Payload architecture}

The block diagram of the PDO-MEMS payload is depicted in Figure 7. This circuit uses a PDO to detect in real time the MEMS oscillation frequency, related to the current mass of the material under test, and sends this information in digital format to the ${ }^{3}$ Cat- 1 central unit. The central unit of the satellite is a PortuxG20 single-board

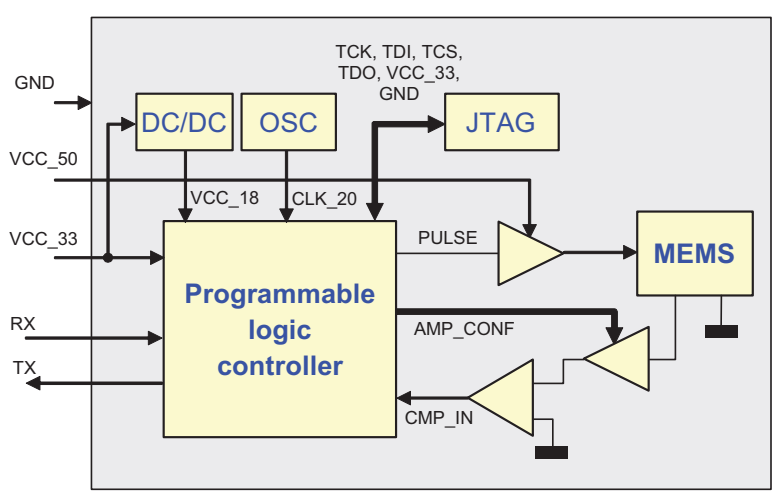

Figure 7: Block diagram of the PDO-MEMS payload.

computer, which includes an ATM91SAM9G20 (ARM9 core) CPU running at $400 \mathrm{MHz}$ with $64 \mathrm{MB}$ of SDRAM and interfaces with the subsystems and payloads through different serial buses.

In order to fulfil the constraints imposed from the mission (e.g. $\leq 0.5 \mathrm{~W}$ of power budget, $\leq 4 \times 4 \mathrm{~cm}^{2}$ of PCB area and $\leq 10 \mathrm{~m}$ of experiment time), the payload was designed as a standalone subsystem made with low power and hardness compliant COTS components, following the "as simple and robust as possible" rule. Only two signals, TX and RX, interface with the central unit. Inputs VCC50 and VCC33 provide $5 \mathrm{~V}$ and $3.3 \mathrm{~V}$ voltage supplies. The MEMS is actuated with $5 \mathrm{~V}$ pulses, whereas a programmable-gain amplification stage and a comparator allow sensing MEMS deflection.

The main component is an EPM240GF100C5N device from Altera. This non-volatile programmable FPGA has $3.3 \mathrm{~V}$ and $1.8 \mathrm{~V}$ inputs, supplied respectively by VCC33 and a DC/DC down converter. The device is clocked by a $20 \mathrm{MHz}$ quartz oscillator and programmed via a JTAG header. The VHDL code implemented includes three main functional modules:

- Clock generator. Provides the PDO sampling signal from the $20 \mathrm{MHz}$ global clock so that $f_{S}$ is configurable between $20 \mathrm{kHz}$ and $5 \mathrm{MHz}$, with 8-bit resolution.

- PDO loop. Configurable parameters are: number of delays (from $L=1$ to 16$)$, loop $\operatorname{sign}\left(0^{\circ}\right.$ or $180^{\circ}$ of phase), voltage/force pulse width (from 50 ns to 6.4 $\mu \mathrm{s}$, in $50 \mathrm{~ns}$ steps) and gain of the sensing amplifier.

- Control. Communicates with the central unit and decodes and executes the commands received, which can be either to test the system, or to configure, run or stop the PDO. When the PDO is running, the module sends the bitstream data to the central unit.

Figure 8 shows a picture of the PCB containing the PDO-MEMS payload. It is placed in the top-outer level of the CubeSat stack, closer to the camera payload. A close- 


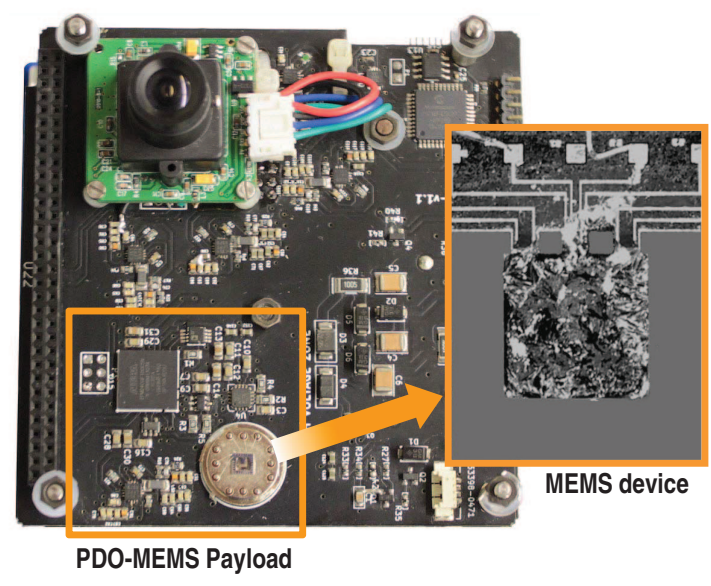

Figure 8: Picture of the top printed circuit board of the ${ }^{3}$ Cat-1, with the position of the PDO-MEMS payload highlighted. A zoom of the MEMS with the deposited pentacene layer is also shown.

view of the MEMS with the Pentacene layer deposited is also shown.

\subsection{On board measurement protocol}

In the mission the operation of the payload is controlled from the central unit of the ${ }^{3}$ Cat- 1 . To save power, the payload is turned off by default and powered only when experiments must be done. Two measurement batches are scheduled daily at the same orbit positions, i.e. in comparable conditions. Figure 9 describes the protocol of a measurement batch, which implies monitoring the PDO oscillation frequency for several configurations, corresponding to different mechanical modes of the MEMS. It consists on the following stages:

1. Start up: a delay of $500 \mathrm{~ms}$ after power up is necessary to initialize the system.

2. Configuration: the PDO is stopped. Then a complete configuration (gain, sampling frequency, pulse width, sign and number of delays) to force oscillation in a specific mode is sent from the central unit.

3. Measurement: the PDO works tuned to the mode configured for $52 \times 2^{16}$ sampling periods. During this time the bitstream data is sent to the central unit in 16-bit words.

4. Iterate - End: stages 2-3 are repeated for 16 different configurations. Then the system is stopped.

In the measurement stage, each data word records the bitstream events produced during $2^{16}$ consecutive sampling periods. Therefore, a measurement batch transmits 1664 data bytes. However, to improve the reliability of the results and also to reduce memory usage, the data corresponding to each iteration is processed in the central unit as follows: 1) the first 4 words are discarded because they correspond to the start up transient of the PDO; 2) the next 48 words are added and accumulated in groups of 24, resulting in two 20-bit data words. Consequently,

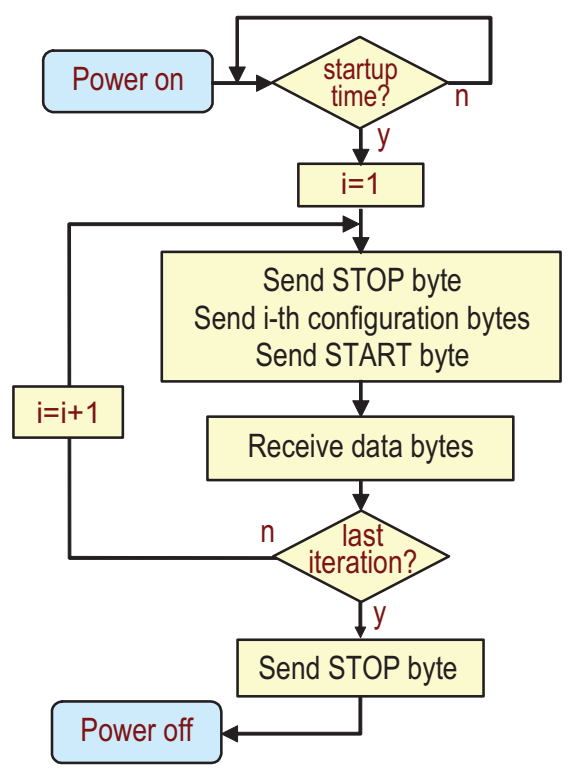

Figure 9: Measurement procedure, as seen from the central unit of the ${ }^{3}$ Cat- 1 .

the result of a measurement batch is stored and sent to ground as 16 iterations/modes, each containing two 20-bit data words plus the current system temperature, date and time.

Let us note here that large temperature variations affect the mechanical performance of MEMS resonators. For instance, frequency shifts of approximately $30 \mathrm{ppm} /{ }^{\circ} \mathrm{C}$ are found in silicon structures [25]. In our case, temperature variations of up to $25^{\circ} \mathrm{C}$ are expected during a complete orbit, therefore it is essential to correlate the results with temperature. To this purpose, the current system temperature for each frequency/mode measurement is also recorded.

The frequency resolution provided by a 16-bit word, obtained by subtracting two consecutive values of $f_{O S C}$ from eq. (6), is $\Delta f_{O S C}=f_{S} / 2^{16}$. Then, assuming a constant oscillation frequency during the iteration time, the resolution after $N \times 2^{16}$ consecutive sampling periods becomes $f_{S} /\left(N \times 2^{16}\right)$. The values selected for the mission are $N=24$ and $f_{S}=110 \mathrm{kHz}$, therefore the frequency resolution of a 20-bit word is $\Delta f_{O S C}=0.07 \mathrm{~Hz}$; for the reference device working around its first mechanical mode, this corresponds to a mass resolution of $0.5 \times 10^{-12} \mathrm{Kg}$. Besides, the duration of a measurement batch is 8.25 minutes and the power consumption is below $125 \mathrm{~mW}$.

\section{Experimental results and discussion}

\subsection{Initial tests}

The objective was to check the functionality of the system designed. A payload prototype, built as a standalone PCB, was used for this purpose. The first tests were made in air conditions and focused on using the PDO to excite 
different mechanical resonances in the reference device. A laser-Doppler vibrometer allowed to measure the movement of the MEMS. The corresponding PDO bitstream data was also recorded.

Up to three mechanical modes could be excited and measured on the reference device. Figure 10 shows the results obtained at each mode from both the vibrometer (3D deflection caption and frequency and amplitude of the resonances) and from the PDO bitstream (frequency spectrum). The frequencies of the first, second and third modes are $3.85,25.24$ and $72.71 \mathrm{kHz}$, respectively. The perfect match between vibrometer and bitstream results validates the suitability of the system. These results also agree with those of Figure 6 .

The same set of experiments was repeated in pressure conditions similar to LEO. The prototype was placed in a high vacuum chamber under $10^{-6}$ mbar of pressure. As expected, damping losses decreased drastically and therefore the oscillation frequencies of all modes increased. The values of the three first resonances, inferred from the bitstream, but also directly measured in an oscilloscope connected to the output of the sensing amplifier, raised up to $4.578,31.132$ and $88.102 \mathrm{kHz}$.

Additionally, modal simulations of the reference MEMS with the Finite-Element-Method software Comsol Multiphysics had been carried out. The frequencies of the first three modes implying vertical deflection in the axis of the device (thus detectable by our system) are in good agreement with the ones measured. This is shown in Table 1 . which summarizes the results of the initial tests.

Table 1: Mechanical modes of the reference device $[\mathrm{kHz}]$ obtained from experiments and FEM simulations.

\begin{tabular}{|r|c|c|c|c|}
\hline $\begin{array}{r}\text { Mech. } \\
\text { mode }\end{array}$ & $\begin{array}{c}\text { Vibr. } \\
\text { air }\end{array}$ & $\begin{array}{c}\text { PDO } \\
\text { air }\end{array}$ & $\begin{array}{c}\text { PDO } \\
\text { vacuum }\end{array}$ & $\begin{array}{c}\text { Comsol } \\
\text { vacuum }\end{array}$ \\
\hline $1^{\text {st }}$ & 3.85 & 3.86 & 4.578 & 4.613 \\
$2^{\text {nd }}$ & 25.24 & 25.24 & 31.132 & 31.094 \\
$3^{\text {rd }}$ & 72.71 & 72.71 & 88.102 & 90.003 \\
\hline
\end{tabular}

\subsection{Pentacene deposition and payload tests}

Having successfully tested the prototype and characterized the reference device, the next step was adding the polymer to the MEMS. To this effect, a $15 \mathrm{mg} / \mathrm{ml}$ Toluene solution of TIPS-Pentacene of $>99.5 \%$ purity (HPLC) was prepared, then deposited on top of the Silicon plate using drop casting techniques with a Teflon probe and finally dried in clean-room ambient. Figure 8 shows a picture of the MEMS with the Pentacene deposited. Due to the deposition technique used, the unique applicable in our case, crystallization and thickness uniformity of the layer are poor.

The amount of Pentacene mass deposited was estimated as follows: 20 drops of solution were deposited on a glass surface following exactly the same procedure and
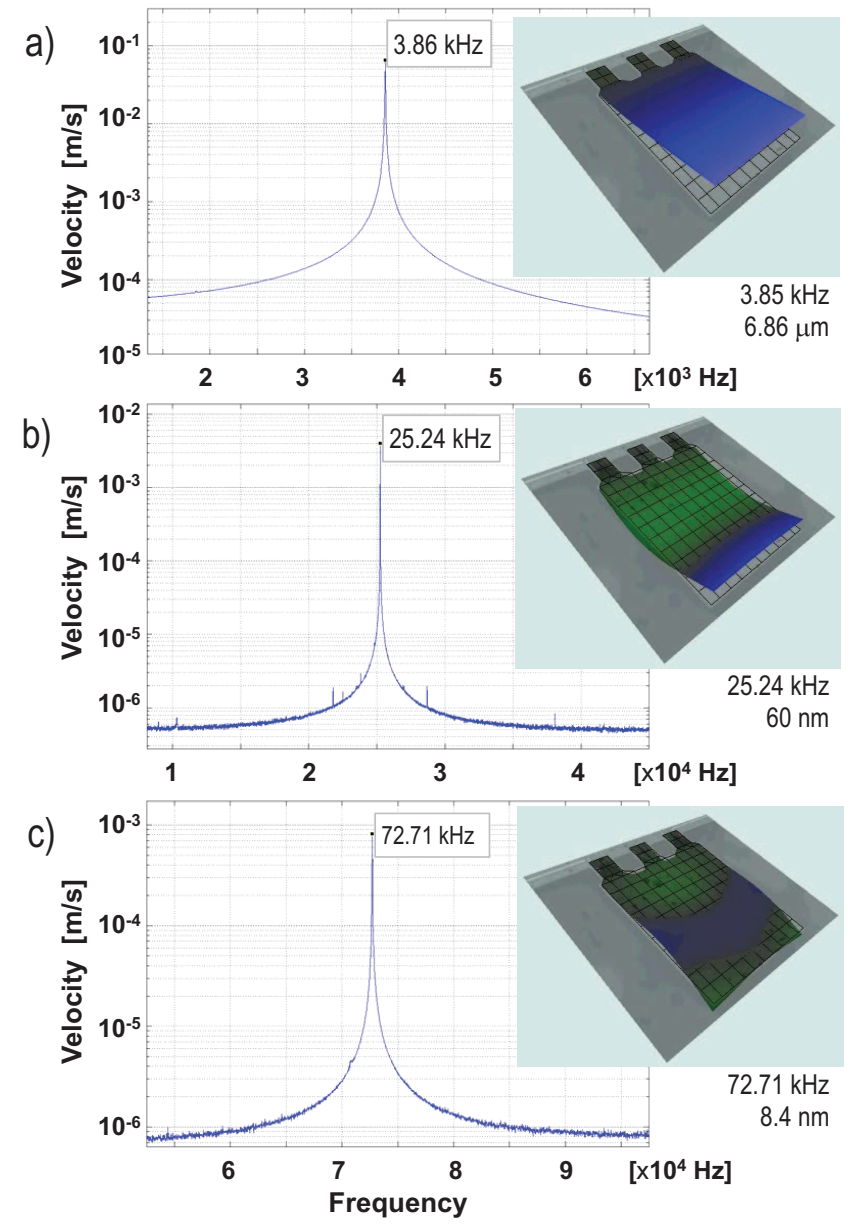

Figure 10: Selective excitation of the first (a), second (b) and third (c) mechanical modes of the MEMS resonator with PDO. For each mode, the bitstream spectrum and the movement caption from a laser-Doppler vibrometer are shown. All measurements are performed in ambient pressure. The PDO sampling frequency is $f_{S}=100 \mathrm{kHz}$.

conditions as with the MEMS. Once the solvent evaporated, a profilometer allowed measuring the volume of each deposited sample and, from it, estimating its mass. The mean value obtained is $3.34 \times 10^{-9} \mathrm{Kg}$ with $38 \%$ of standard deviation.

The next experiments were performed with the final version of the payload, once it passed the qualification tests. In this case the objective was to check the features of the entire system after Pentacene deposition. The ${ }^{3}$ Cat- 1 PCB was placed inside the high vacuum chamber and the measurement protocol described in Figure 9] was applied, processing the bitstream data as explained in Section 3.4 above.

As expected, the mass added by the Pentacene layer produced notable decreases in the frequencies measured. The first mode resonance was found at $4.098 \mathrm{kHz}$, whereas the second was at $28.686 \mathrm{kHz}$. The third mode provided unstable values. Assuming a mass increase of $3.34 \times 10^{-9}$ $\mathrm{Kg}$, these results imply a sensitivity around $6.58 \times 10^{-12}$ 
$\mathrm{Kg} / \mathrm{Hz}$ for the first mode and around $1.36 \times 10^{-12} \mathrm{Kg} / \mathrm{Hz}$ for the second mode.

Comsol FEM simulations were also performed to check consistence with the experimental results. In the simulations, a uniform thickness of $2.75 \mu \mathrm{m}$ was assumed for the deposited layer, which for the TIPS-Pentacene density, $1.104 \mathrm{Kg} / \mathrm{l}$, yields the same mass increase considered above. As shown in Table 2 the frequency values obtained for the two first two modes are in good agreement with their experimental counterparts.

Table 2: Mechanical modes of the reference device after Pentacene deposition $[\mathrm{kHz}]$ in high vacuum obtained from experiments and FEM simulations. Frequency decreases due to the Pentacene deposition are also included.

\begin{tabular}{|r|c|c|c|c|}
\hline $\begin{array}{r}\text { Mech. } \\
\text { mode }\end{array}$ & $\begin{array}{c}\text { PDO } \\
f_{O S C}\end{array}$ & $\begin{array}{c}\text { PDO } \\
f_{\text {shift }}\end{array}$ & $\begin{array}{c}\text { Comsol } \\
f_{\text {OSC }}\end{array}$ & $\begin{array}{c}\text { Comsol } \\
f_{\text {shift }}\end{array}$ \\
\hline $1^{\text {st }}$ & 4.098 & -0.480 & 4.110 & -0.503 \\
$2^{\text {nd }}$ & 28.686 & -2.446 & 28.086 & -3.008 \\
$3^{\text {rd }}$ & - & - & 81.692 & -8.311 \\
\hline
\end{tabular}

\section{Conclusions}

A new method to in-situ sense the degradation of a polymer due to atomic oxygen erosion in low earth orbit has been presented. It provides real-time information on how the degradation occurs, eliminating the need to work with samples recovered once the mission has finished. The method has been implemented as a CubeSat payload.

The detection principle is mass sensing using a MEMS resonator: polymer mass variations produce shifts in the mechanical oscillation frequency of the MEMS. The MEMS is embedded in a pulsed digital oscillator, a kind of circuit that directly provides the oscillation frequency in digital format. This circuit allows exciting different mechanical modes of the MEMS. A polymer of interest for emerging electronic applications, TIPS-Pentacene, was chosen.

The payload is a simple, re-configurable and robust design made with COTS components. It met the power, area and timing requirements of the mission and passed the qualification tests. The MEMS resonator was characterized using both a pulsed digital oscillator, a vibrometer equipment and FEM simulations. Complete ground tests of the payload were performed before and after polymer deposition. The results indicate that polymer mass losses around $10^{-12} \mathrm{Kg}$ can be detected successfully, with sensitivities of $6.58 \times 10^{-12} \mathrm{Kg} / \mathrm{Hz}$ for the first and $1.36 \times 10^{-12}$ $\mathrm{Kg} / \mathrm{Hz}$ for the second mechanical mode of the MEMS.

Acknowledgements: This work was supported in part by the Spanish Ministry MINECO under Project TEC201348102-C2-1-P.

\section{References}

[1] A. de Rooij, Encyclopedia of Aerospace Engineering, John Wiley and Sons Ltd, 2010, Ch. Corrosion in space.

[2] S.K.R. Miller, B. Banks, Degradation of spacecraft materials in the space environment, MRS Bulletin 35 (1) (2010) 20-24.

[3] L. Leger, Oxygen atom reaction with shuttle materials at orbital altitudes, Tech. Rep. 58246, NASA (1982).

[4] E. Silverman, Space environmental effects on spacecraft LEO materials selection guide, Tech. Rep. 4661, NASA (1995).

[5] K.K. De Groh, B.A. Banks, C.E. Mccarthy, R.N. Rucker, L.M. Roberts, L.A. Berger, LEO MISSE 2 PEACE Polymers Atomic Oxygen Erosion Experiment on the International Space Station, High Performance Polymers 20 (4-5) (2008) 388-409.

[6] B.A. Banks, J.A. Backus, M.V. Manno, D.L. Waters, K.C. Cameron, K.K. de Groh, Atomic oxygen erosion yield prediction for spacecraft polymers in low earth orbit, in: 11th Int. Symp. on Materials in the Space Environment (ISME-11), Sept. 2009.

[7] S.K.R. Miller, B.A. Banks, D.L. Waters, Investigation into the differences between atomic oxygen erosion yields of materials in ground-based facilities and LEO, High Performance Polymers 20 (4-5) (2008) 523-534.

[8] M. Tagawa, K. Yokota, Problems and recent trends on material degradation studies in a real and simulated space environment, in: Proceedings of the 5th Spacecraft Environment Symp. JAXA, 2009, pp. 66-70.

[9] P. Ehrenfreund, et al., The O/OREOS mission-astrobiology in low earth orbit, Acta Astronautica 93 (2014) 501-508.

[10] D. Noack, K. Brieß, Laboratory investigation of a fluid-dynamic actuator designed for cubesats, Acta Astronautica 96 (2014) 7882.

[11] B. Rievers, A. Milke, D. Salden, Cubesat in-situ degradation detector (CIDD), Acta Astronautica 112 (2015) 69-76.

[12] R. Jove-Casulleras, Contribution to the development of picosatellites for earth observation and technology demonstrators, Ph.D. thesis, Universitat Politecnica de Catalunya BarcelonaTech, http://hdl.handle.net/10803/286237 (2015).

[13] A. Vollmer, H. Weiss, S. Rentenberger, I. Salzmann, J.P. Rabe, N. Koch, The interaction of oxygen and ozone with pentacene, Surface Science 600 (2006) 2004-2007.

[14] W. Fudickar, T. Linker, Why triple bonds protect acenes from oxidation and decomposition, Journal of the American Chemical Society 134 (2012) 15071-15082.

[15] S.K. Park, D.A. Mourey, J.I. Han, J.E. Anthony, T.N. Jackson, Environmental and operational stability of solution-processed 6,13-bis (triisopropylsilylethynyl) pentacene thin film transistors, Organic Electronics 10 (3) (2009) 486-1490.

[16] M. Domínguez-Pumar, J. Pons-Nin, J. Ricart, General dynamics of pulsed digital oscillators, IEEE Trans. on Circuits and Systems I 55 (7) (2008) 2038-2050.

[17] J. Ricart, J. Pons-Nin, M. Dominguez-Pumar, A. Rodriguez, E. Figueras, M. Horrillo, J. Gutierrez, I. Sayago, Application of pulsed digital oscillators to volatile organic compounds sensing, Sensors and Actuators B: Chemical 134 (2) (2008) 773-779.

[18] J. Ricart, J. Pons-Nin, S. Gorreta, M. Dominguez-Pumar, Application of pulsed digital oscillators to the in-situ testing of MEMS vacuum packaging, in: 14th Int. Conf. on Sensors, Technologies, Electronics and Applications (SENSOR 2009), Nurnberg, Germany, May 2009.

[19] S. R. Norsworthy, R. Schreier, G. C. Temes, et al., Delta-sigma data converters: theory, design, and simulation, Vol. 97, IEEE press New York, 1996.

[20] E. Blokhina, J. Pons-Nin, J. Ricart, O. Feely, M. DominguezPumar, Control of MEMS vibration modes with pulsed digital oscillators. part I - theory, IEEE Trans. on Circuits and Systems I 57 (8) (2010) 1865-1878.

[21] J. Ricart, J. Pons-Nin, E. Blokhina, S. Gorreta, J. Hernando, T. Manzaneque, J. Sanchez-Rojas, O. Feely, M. DominguezPumar, Control of MEMS vibration modes with pulsed digital oscillators. part II - simulation and experimental results, IEEE Trans. on Circuits and Systems I 57 (8) (2010) 1879-1890. 
[22] E. Bou-Balust, A. Hu, E. Alarcon, Scalability analysis of SIMO non-radiative resonant wireless power transfer systems based on circuit models, IEEE Trans. on Circuits and Systems I 62 (10) (2015) 2574-2583.

[23] P. Ortega, R. Jove-Casulleras, A. Pedret, R. Gonzalvez, G. Lopez, I. Martın, M. Domínguez, R. Alcubilla, A. Camps, An IBC solar cell for the UPCSat-1 mission, in: Proc. of the 9th Spanish Conf. on Electron Devices (CDE 2013), 2013.

[24] R. Jove-Casulleras, J. Ramos, H. Ccorimanya, A. Camps, A. Amezaga, E. Alarcon, E. Bou, Temperature gradient sensor from pulsed power supply duty cycle in ultra-low-power energy harvesting system, Electronics Letters 50 (11) (2014) 826-828.

[25] E. Iervolino, M. Riccio, A.W. van Herwaarden, A. Irace, G. Breglio, W. van der Vlist, P.M. Sarro, Temperature dependence of the resonance frequency of thermogravimetric devices, in: Proc. Eurosensors XXIV, Sept. 2010. 